\title{
In vitro- exploration of fungal endophytes of Egyptian Cynara scolymus L. (artichoke) and investigation of some their bioactive potentials
}

\author{
Nermien H.S. ${ }^{1,2,}{ }^{*}$, Thabet A. ${ }^{3,4}$, Markeb A.A. ${ }^{3}$, Sayed D.M. ${ }^{5}$ and El-Maali N.A. ${ }^{3}$ \\ ${ }^{1}$ Department of Botany and Microbiology, Faculty of Science, Assiut University Assiut 71516, Egypt \\ ${ }^{2}$ College of Applied Medical Sciences - Jubail, Imam Abdulrahman Bin Faisal University, Dammam, KSA \\ ${ }^{3}$ Department of Chemistry, Faculty of Science, Assiut University, Assiut 71516, Egypt \\ ${ }^{4}$ Department of Chemistry, Faculty of Science, Sana'a University, Sana'a, Yemen \\ ${ }^{5}$ Department of Oncological Clinical Pathology, South Egypt Cancer Institute, Assiut University, Assiut 71516, Egypt \\ Received: 23/07/2018, Accepted: 09/11/2018, Available online: 21/11/2018 \\ *to whom all correspondence should be addressed: e-mail: nermienhelmyseddek@gmail.com, nhsali@iau.edu.sa \\ https://doi.org/10.30955/gnj.002836
}

\begin{abstract}
Plant-associated microorganisms, especially endophytic fungi, represent an untapped resource for the discovery of biologically active natural products. The objectives of this study were to isolate, identify endophytic fungi, and produce their bioactive metabolites from the leaves of two varieties of Egyptian artichoke namely: French Hyrious and Egyptian Baladi. In addition, assess of their total antioxidant capacity (TAC), total phenolic content (TPC) and total flavonoid content (TFC). The results of this novel study show a total of 35 endophytic fungal species belonging to 14 genera were isolated from both artichoke leaves with gross total counts of colonizing endophytic fungi ranged from 71 to $123 \mathrm{cfu}$ which is matching $78.89 \%$ to $136.67 \%$ of colonization frequency. All taxa recovered were assigned to Ascomycetes. In addition, there is high species richness and diversity indices of endophytic filamentous fungi in the leaves Baladi Artichoke as compared to its French rival. Alternaria alternata were found to be the most frequently isolated dominant species. The TAC, TPC and TFC of the fungal cultures ranged from 163 to $681 \mathrm{mgAAE} / \mathrm{gDW}, 10.38$ to $40.30 \mathrm{mgGAE} / \mathrm{gDW}$, and 13.92 to $173.55 \mathrm{mgQE} / \mathrm{gDW}$, respectively. Furthermore, LC-ESI-MS/MS confirmed the presence of 1,3-dicaffeoylquinic acid and 1,5-dicaffeoylquinic acid in the methanolic extract of $A$. alternata. Hence, this novel study suggested that the metabolites produced by endophytic fungi associated with Egyptian artichoke could be explored as an economic and potential natural resources with diverse pharmaceutical and biological activities.
\end{abstract}

Keywords: Artichoke, fungal endophytes, antioxidant capacity, total flavonoids, total phenolics contents.

\section{Introduction}

As an alternative to drug therapy, it is desirable to use foods, food components, and/or medicinal plants for the prevention and treatment of different diseases and thereby avoid the adverse effects of organically synthesized drugs as well as the high-cost drug therapy (Roghani-Dehkordi and Kamkhah, 2009). Medicinal plants are the richest bio-resource of drugs from traditional systems and modern medicines, nutraceuticals, food supplements, folk medicines, pharmaceutical intermediates and chemical entities for synthetic drugs. World Health Organization (WHO) encourages, advises and promotes herbal remedies in national health care programmers due to the low cost of these drugs (Amita Pandey, 2014). Recently, medicinal plants have received the attention of the pharmaceutical and scientific societies. The biomolecules of pharmaceutical and nutraceutical interest are mainly represented by flavonoids and phenolic acids, particularly caffeic acid and its derivates mono- and bi-caffeoylquinic acids (Saez et al., 2013). Recent studies have reported that bioactive phenolic constituents from plants and endophytic microbe have a wide variety of biological activities, such as antioxidants, hepatoprotective, anti-inflammatory, antifibrosis, and antiviral activities, and anticarcinogenic effects against different cancer cell line (Chutulo and Chalannavar, 2018; Gebhardt and Fausel, 1997; Liang et al., 2012; Llorach et al., 2002; Sihem DABBOU et al., 2017; Simona Spînu et al., 2017; Wang et al., 2014). Also, Plant-derived bioactive compounds have achieved an extensive interest due to their versatile applications and beneficial effects; for instance, antioxidant activity, digestive stimulation action, anti-inflammatory, antimicrobial, hypolipidemic, antimutagenic effects and anticarcinogenic anticarcinogenic potential (Jacociunas et al., 2013; Wojdylo et al., 2007), on health promotion because the low side effects in comparison to the synthetic drugs (Farag et al., 2013; Ncube and Afolayan, 2008). Th. Thus, many publications recorded its medicinal values as natural compounds. 
Artichoke (Cynara scolymus L.) is an ancient herbaceous perennial plant species belonging to the Asteraceae family, genus Cynara (Farag et al., 2013; Xia et al., 2014), originating from the Mediterranean region, and it is now cultivated worldwide. According to FAO estimation, Italy was the world's largest artichoke producer in 2016 followed by Egypt, Spain Peru (FAO, 2016). The artichoke was used as food and medicine by ancient Egyptians, Greeks, and Romans. Three Cynara scolymus (artichoke) cultivars: American Green Globe, French Hyrious, and Egyptian Baladi have been cultivated in the Northern part of Egypt (Farag et al., 2013). Moreover, the artichoke is one of the oldest medicinal plants with multiple health benefits (Xia et al., 2014). Artichoke has many natural antioxidants and multiple pharmacological actions. The antioxidant properties of artichoke are thought to be related to its abundant phenolic composition (El Morsy and Kamel, 2014). Recent studies have reported that Artichoke has antitoxic activity (Heidarian and Rafieian-Kopaei, 2013), cholesterol-reducing effect (Kusku-Kiraz et al., 2010) anticarcinogenic, antigenotoxic, hepatoprotective, bile expelling, diuretic, and antiinflammatory, as well as anti-HIV, antifungal, and antibacterial (Abu-Reidah et al., 2013). Additionally, its leaves are used in traditional medicine as an herbal medicament for the treatment of hepatitis, choleretic, diuretic, hyperlipidemia, obesity and dyspeptic disorders (Nassar et al., 2013). Artichoke was ranked fourth out of more than 1000 food products in antioxidant content, and the first among several selected vegetable crops (Yoo et al., 2012). Artichoke by-products (leaves, external bracts and stems) represent an enormous amount of discarded materials (representing $80-85 \%$ of the biomass) (Lattanzio et al., 2009). Recently, the possibility to recover them has been proposed for economic, environmental concerns, adding value to agro-industrial by-products (Ruiz-Cano et al., 2014).

Endophytic fungi residing internally in the healthy living plants tissues without causing any immediate harm to their host (Yadav et al., 2014). All higher plants host one or more endophytic microbe on this green planet (Strobel and Daisy, 2003) from the arctic to the tropics. In recent years, endophytic fungi have been received great attention because they have the ability to benefit host plant growth. The research on endophytes showed that they are obviously a rich and reliable source of bioactive secondary metabolites and chemically novel compounds with huge medicinal, agricultural potential, and industry (Yi-Shuan Chen et al., 2015). Endophytic fungi and their associated higher plants are shown to be a good source of novel antioxidants (Sandhu and Gupta, 2015). The endophytic fungi seem to yield bioactive compounds, originally isolated from their host plants, as well as bioactive metabolites that are clearly different from those of plants and feature unique structural characteristics, which may have potential use in agriculture and medicine (Silva-Hughes et al., 2015; Stierle et al., 1995; Wang et al., 2013). In addition, it is well known as a microbial source of a valuable products and it is generally easier and more economically to produce (Chen et al., 2010). Metabolites isolated from the fungal endophytes are good sources of novel secondary metabolic products possess diverse and unique structural groups (Chutulo and Chalannavar, 2018; Schulz et al., 2002; Sharma et al., 2016). Numerous work has been focused on the bioactive potential of endophytes, such as antiviral, antibacterial, antifungal, anticancer, antiviral, insecticide, antidiabetic and immunosuppressive activities, but few is known about their antioxidant capacity (Yadav et al., 2014). Shukla et al. was isolating endophytic fungi from Ocimum sanctum and showing their antioxidant activity. A strong antioxidant activity was found in the endophytic fungus of Phyllosticta sp. isolated from Guazumato mentos plant. Therefore, by fermentation an endophytic fungus isolated from Artichoke (Cynara scolymus L.) can produce bioactive compounds such as chlorogenic acid, caffeoylquinic acids, caffeic acid and its derivatives (apigenin and luteolin) just like its host plant. This will protect the plant from extinction. Thus, an immense opportunity to discover novel natural products from hitherto less investigated fungal endophytes that reside in hitherto assumed medicinal plants. Also, microbial natural products represent a huge and largely important resource of unique chemical structures and probably play an important role for adaptation as a response to habitat (Gunatilaka, 2006).

Based on this theory, the aim of this study is: (1) to isolate and identify each endophytic fungus from two artichoke cultivars (French Hyrious and Egyptian Baladi), (2) to investigate the total polyphenols and the total flavonoids content and (3) to evaluate the antioxidant activities using in vitro study of the Methanolic crude extracts of C. scolymus L. and its fungal endophytes. To the best of our knowledge, no previous work on fungal endophytes and its metabolites investigated from Artichoke plant, so this research considered a new record on this investigation. By implementation of our present work, it becomes easier, cheaper and time consuming to get the bioactive compounds (like flavonoids, phenolic compound, anti-microbial or anti-cancer compounds) of plants by their endophytic fungi via fermentation processes. Additionally, we open the way for all to discover novel endophytes isolated from new medicinal plants to get novel bioactive compound. Also, that is invitation for preserving medicinal plants by decrease plant uprooting and use their endophytes to improve environmental science research.

\section{Materials and methods}

\subsection{Chemicals and reagents}

Aluminum chloride, sodium acetate, sulfuric acid, Folin-Ciocalteu (FC) reagent, gallic acid (GAE), ascorbic acid (AA), quercetin (QE), luteolin-7-O-rutinoside, 1,3-dicaffeoylquinic acid (1,3-diCQA), 1,5-dicaffeoylquinic acid (1,5-diCQA), chlorogenic acid, caffeic acid, ethanol, methanol and formic acid of analytical or HPLC grade were purchased from Sigma Aldrich. Ammonium molybdate was purchased from Fluka Co. (Bushes, Switzerland). Assay of all standards, solvents, and 
reagents were $\geq 99 \%$. Water was purified by using a Milli-Q system.

\subsection{Plant material}

Two Artichoke varieties, Cynara scolymus L, cultivars namely; Egyptian Baladi and French Hyrious, were collected from Markaz Abu Al Matamir, Beheira governorate, Egypt, during February-April 2015. The collected plants have been confirmed by plant taxonomists at Botany and Microbiology Department, Faculty of Science, Assiut University, 71516-Assiut, Egypt.

\subsection{Evaluation for presence of fungal endophytes}

\subsubsection{Surface sterilization}

To ascertain the possible presence of the fungus in the internal plant tissues, one hundred separate healthy artichoke plants were collected from the field, but we choose 10 samples randomly for investigation. From each sample, three leaves were randomly selected and individually washed thoroughly in running tap water to remove dust and debris then surface-disinfected by washing with $70 \%$ ethanol for 2 min, submersing in $5-6 \%$ sodium hypochlorite for $3 \mathrm{~min}$ then $70 \%$ ethanol for $30 \mathrm{sec}$. respectively, followed by two rinses in sterile autoclaved distilled water and finally were dried between sterilized filter paper (Arnold et al., 2001). Foliar leaves were cut using a sterile scalpel into $1 \mathrm{~cm}^{2}$ in diameter with and without midrib. Then, three leaf segments were inserted in sterilized petri dishes $(9 \mathrm{~cm}$ diameter) individually containing sterile media, replicated three times. All Petri plates were incubated at $28 \pm 2{ }^{\circ} \mathrm{C}$ in darkness for 10-15 days.

\subsubsection{Used cultivated media}

Three different types of media were used in this investigation namely; potato dextrose agar (PDA), Glucose- Czapek's (CZ) and Cellulose-Czapek's (C) agar media (Smith and Onions, 1994). To count and collect all filamentous fungal endophytes which can grow on this media and was expected in studied leaves.

\subsubsection{Efficacy of surface sterilization}

To confirm the sterilization, the final rinse water was plated to determine whether the sterilization process was successful in eliminating epiphytic microorganisms. Also, another sterilized leaf segments (10 gm) were placed in a conical flask containing $200 \mathrm{~mL}$ sterile distilled water, the suspension was shaken by a mechanical shaker at $150 \mathrm{rpm}$ for 10 minutes. One $\mathrm{mL}$ of this dilution (1/200) was transferred aseptically into five replicates of sterilized Petri dishes and 12-15 mL of PDA agar medium, cooled to just above the solidifying temperature, were added to each dish. The dishes were rotated by hand for good dispersion. The absence of growth of any fungi on the all Petri dishes confirmed that the surface sterilization procedure was effective (Schulz et al., 1993). All Petri plates were incubated at $28 \pm 2{ }^{\circ} \mathrm{C}$ in darkness for 10-15 days.

\subsubsection{Phenotypic identification of fungal endophytes}

Periodically the colonies were examined, counted and each colony that emerged from segments was transferred to antibiotic-free Potato Dextrose Agar medium (PDA) to aid macroscopic and microscopic identification using morphological criteria of fruiting body and spores. Standard taxonomic manuals were used to identify the fungal genera. Any fungal growth was sub-cultured on individual plates containing PDA for preservation at Multidisciplinary Research Center (MRCE), Assiut University for further work. The percentage of fungal endophytes present in the tissues sampled was assessed.

\subsubsection{Calculation of colonization frequency}

Colonization frequency (CF), \%, of an endophyte species was calculated as reported (Pandey et al., 2003; Suryanarayanan, 2003).

$\mathrm{CF} \%=\mathrm{N}_{\text {col }} / \mathrm{N}_{\mathrm{t}} \times 100$

Where: $N_{\text {col }}$ is the number of segments colonized by a single endophyte, and $\mathrm{N}_{\mathrm{t}}$ is the total number of segments examined.

\subsection{Preparation of fungal extracts}

Fungal extracts were prepared according to the reported method with slight modifications (Sutjaritvorakul, 2011). Briefly, $1 \mathrm{~cm}^{2}$ disc of 7-day-old colony of each tested fungal isolated was inoculated into $250 \mathrm{~mL}$ Erlenmeyer conical flask containing $150 \mathrm{~mL}$ potato dextrose broth (PDB) media. Cultures of colonies were incubated at $120 \mathrm{rpm}$ and $28 \pm 2{ }^{\circ} \mathrm{C}$ for 10 days. Next, the growing mycelia were extracted using methanol: PDB (2: 1) and homogenized at $16000 \mathrm{rpm}$. Then, the methanol extracts were filtered through filter paper No. 1 and the filtrates were evaporated under reduced pressure at $40{ }^{\circ} \mathrm{C}$ till dryness. Finally, the obtained residues were stored at $-20{ }^{\circ} \mathrm{C}$ for further investigations.

\subsection{Total phenolics content}

The total phenolics content (TPC) of all extracts was performed using the Folin-Ciocalteu (FC) reagent with slight modifications of the reported method in the literature (Ainsworth and Gillespie, 2007). Initially, $300 \mu \mathrm{L}$ of methanolic extracts, or gallic acid (GA) standard in methanol were mixed well with $0.6 \mathrm{~mL}$ of $10 \%(\mathrm{v} / \mathrm{v}) \mathrm{FC}$ reagent, then vortexed thoroughly for $10 \mathrm{sec}$, and incubated at room temperature for $3 \mathrm{~min}$. After the addition of $2.4 \mathrm{~mL}$ of $700 \mathrm{mM} \mathrm{Na} \mathrm{CO}_{3}$ to the mixture, followed by vortexing, covered and incubating for $2 \mathrm{~h}$ at room temperature, the absorbance was measured at 765 $\mathrm{nm}$ using a Cary 60, Agilent technologies, UV/VIS spectrophotometer. The TPC was expressed as mggallic acid equivalent (GAE) per $g$ of plant dry weight (mgGAE/gDW). A calibration curve was constructed by using series of calibration standards of GA in methanol ( 1 , $15.62,31.5,62.5,125,250$ and $500 \mathrm{mg} / \mathrm{L}$ ). All tests were carried out in triplicate and the results were averaged. 
Table 1. Total count (TC), percentage of total count (\%TC), percentage of colonization frequency (\%CF), number of cases of isolation ( $\mathrm{NCl}$; out of 10 samples), and occurrence remark $\left(\mathrm{OR}^{*}\right)$, of endophytic fungi isolated from Egyptian Baladi Artichoke fresh leaves per 90 segments on the three types of media (PDA, CZ, \& cellulose)

\begin{tabular}{|c|c|c|c|c|c|c|c|c|c|c|c|c|c|c|c|}
\hline Types of media & & & PDA & & & & & CZ & & & & & C & & \\
\hline Endophytic fungi & Tc & \%Tc & $\% C F$ & $\mathrm{NCl}$ & OR & Tc & \%Tc & \%CF & $\mathrm{NCl}$ & OR & Tc & \%Tc & \%CF & $\mathrm{NCl}$ & OR \\
\hline Alternaria & 58 & 55.24 & 64.44 & 10 & $\mathrm{H}$ & 36 & 37.89 & 40 & 10 & $\mathrm{H}$ & 52 & 42.28 & 57.78 & 10 & $\mathrm{H}$ \\
\hline $\begin{array}{l}\text { A. alternata (Fr.) } \\
\text { Keiss }\end{array}$ & 51 & 48.57 & 56.67 & 10 & $\mathrm{H}$ & 30 & 31.58 & 33.33 & 10 & $\mathrm{H}$ & 48 & 39.02 & 53.33 & 10 & $\mathrm{H}$ \\
\hline $\begin{array}{c}\text { A. atra (Preuss) } \\
\text { Woudenb. \& } \\
\text { Crous }\end{array}$ & & & & & & 1 & 1.05 & 1.11 & 1 & $\mathrm{~L}$ & & & & & \\
\hline $\begin{array}{l}\text { A. cheiranthi } \\
\text { (Lib.) P.C. Bolle }\end{array}$ & 2 & 1.90 & 2.22 & 2 & $\mathrm{~L}$ & & & & & & & & & & \\
\hline $\begin{array}{c}\text { A. } \\
\text { chlamydospora } \\
\text { Mouch }\end{array}$ & 2 & 1.90 & 2.22 & 1 & $\mathrm{~L}$ & 2 & 2.11 & 2.22 & 1 & $L$ & 1 & 0.81 & 1.11 & 1 & $\mathrm{~L}$ \\
\hline $\begin{array}{l}\text { A. graminicola } \\
\text { E.G. Simmons } s p\end{array}$ & 1 & 0.95 & 1.11 & 1 & $\mathrm{~L}$ & & & & & & & & & & \\
\hline $\begin{array}{l}\text { A. citri Ellis \& } N . \\
\text { Pierce }\end{array}$ & 1 & 0.95 & 1.11 & 1 & L & 2 & 2.11 & 2.22 & 2 & L & & & & & \\
\hline A. mali Roberts & & & & & & & & & & & 1 & 0.81 & 1.11 & 1 & $\mathrm{~L}$ \\
\hline $\begin{array}{c}\text { A. tenuissima } \\
\text { (Kunze) Wiltshire }\end{array}$ & 1 & 0.95 & 1.11 & 1 & $\mathrm{~L}$ & & & & & & 2 & 1.63 & 2.22 & 2 & $\mathrm{~L}$ \\
\hline $\begin{array}{c}\text { A. phragmospora } \\
\text { Emden }\end{array}$ & & & & & & 1 & 1.05 & 1.11 & 1 & $L$ & & & & & \\
\hline Aspergillus & 11 & 10.48 & 12.22 & 6 & $\mathrm{H}$ & 14 & 14.74 & 15.56 & 8 & $\mathrm{H}$ & 19 & 15.45 & 21.11 & 4 & $\mathrm{M}$ \\
\hline A. flavus Link & & & & & & 4 & 4.21 & 4.44 & 3 & $\mathrm{~L}$ & & & & & \\
\hline A. niger Tiegh & 10 & 9.52 & 11.11 & 5 & $\mathrm{M}$ & 10 & 10.53 & 11.11 & 5 & $M$ & 9 & 7.32 & 10.00 & 4 & $M$ \\
\hline A. terreus Thom & 1 & 0.95 & 1.11 & 1 & $\mathrm{~L}$ & & & & & & 10 & 8.13 & 11.11 & 3 & $\mathrm{~L}$ \\
\hline $\begin{array}{l}\text { Chaetomium } \\
\text { globosum Kunze }\end{array}$ & 1 & 0.95 & 1.11 & 1 & $L$ & 1 & 1.05 & 1.11 & 1 & L & 4 & 3.25 & 4.44 & 2 & L \\
\hline Cladosporium & & & & & & 4 & 4.21 & 4.44 & 4 & $M$ & 4 & 3.25 & 4.44 & 1 & $\mathrm{~L}$ \\
\hline $\begin{array}{c}\text { C. } \\
\text { cladosporioides } \\
\text { (Fresen.) G.A. de } \\
\text { Vries }\end{array}$ & & & & & & 2 & 2.11 & 2.22 & 2 & $\mathrm{~L}$ & 4 & 3.25 & 4.44 & 1 & $\mathrm{~L}$ \\
\hline $\begin{array}{c}\text { C. herbarum } \\
\text { (Pers.) Link }\end{array}$ & & & & & & 1 & 1.05 & 1.11 & 1 & $L$ & & & & & \\
\hline $\begin{array}{c}\text { C. } \\
\text { sphaerospermum } \\
\text { Penz }\end{array}$ & & & & & & 1 & 1.05 & 1.11 & 1 & $\mathrm{~L}$ & & & & & \\
\hline $\begin{array}{c}\text { Curvularia } \\
\text { australiensis } \\
\text { (M.B. Ellis) }\end{array}$ & 1 & 0.95 & 1.11 & 1 & $\mathrm{~L}$ & & & & & & & & & & \\
\hline $\begin{array}{l}\text { Epicoccum } \\
\text { nigrum Link }\end{array}$ & & & & & & & & & & & 4 & 3.25 & 4.44 & 4 & $M$ \\
\hline $\begin{array}{c}\text { Fusarium } \\
\text { fujikuroi } \\
\text { Nirenberg }\end{array}$ & & & & & & 4 & 4.21 & 4.44 & 3 & $\mathrm{~L}$ & & & & & \\
\hline $\begin{array}{c}\text { Nigrospora } \\
\text { oryzae (Berk. \& } \\
\text { Broome) Petch }\end{array}$ & 15 & 14.29 & 16.67 & 4 & $M$ & 20 & 21.05 & 22.22 & 9 & $\mathrm{H}$ & 19 & 15.45 & 21.11 & 5 & $M$ \\
\hline Penicillium & 5 & 4.76 & 5.56 & 3 & $\mathrm{~L}$ & 4 & 4.21 & 4.44 & 3 & $\mathrm{~L}$ & 3 & 2.44 & 3.33 & 2 & $\mathrm{~L}$ \\
\hline P. citrinum Thom & 3 & 2.86 & 3.33 & 2 & $\mathrm{~L}$ & 1 & 1.05 & 1.11 & 1 & $\mathrm{~L}$ & & & & & \\
\hline $\begin{array}{l}\text { p. glabrum } \\
\text { (Wehmer) } \\
\text { Westling }\end{array}$ & & & & & & 3 & 3.16 & 3.33 & 2 & $\mathrm{~L}$ & 3 & 2.44 & 3.33 & 2 & $\mathrm{~L}$ \\
\hline
\end{tabular}




\begin{tabular}{|c|c|c|c|c|c|c|c|c|c|c|c|c|c|c|c|}
\hline Types of media & & & PDA & & & & & $\mathbf{C Z}$ & & & & & C & & \\
\hline Endophytic fungi & Tc & \%Tc & $\% \mathrm{CF}$ & $\mathrm{NCl}$ & OR & Tc & \%Tc & \%CF & $\mathrm{NCl}$ & OR & Tc & \%Tc & $\% \mathrm{CF}$ & $\mathrm{NCl}$ & OR \\
\hline $\begin{array}{l}\text { P. fasciculatum } \\
\text { Sommerf. }\end{array}$ & 2 & 1.90 & 2.22 & 1 & $\mathrm{~L}$ & & & & & & & & & & \\
\hline $\begin{array}{c}\text { Stachybotrys } \\
\text { chartarum } \\
\text { (Ehrenb.) S. } \\
\text { Hughes } \\
\end{array}$ & 1 & 0.95 & 1.11 & 1 & $\mathrm{~L}$ & 2 & 2.11 & 2.22 & 1 & L & 1 & 0.81 & 1.11 & 1 & $\mathrm{~L}$ \\
\hline Stemphylium & 6 & 5.71 & 6.67 & 4 & $M$ & 10 & 10.53 & 11.11 & 7 & $\mathrm{H}$ & 15 & 12.20 & 16.67 & 6 & $\mathrm{H}$ \\
\hline $\begin{array}{c}\text { S. botryosum } \\
\text { Wallr }\end{array}$ & 5 & 4.76 & 5.56 & 3 & L & 5 & 5.26 & 5.56 & 3 & $M$ & 3 & 2.44 & 3.33 & 1 & L \\
\hline $\begin{array}{c}\text { S. vesicarium } \\
\text { (Wallr.) E.G. } \\
\text { Simmons }\end{array}$ & & & & & & & & & & & 5 & 4.07 & 5.56 & 2 & $\mathrm{~L}$ \\
\hline Stemphylium sp. & 1 & 0.95 & 1.11 & 1 & $\mathrm{~L}$ & 5 & 5.26 & 5.56 & 4 & $\mathrm{M}$ & 7 & 5.69 & 7.78 & 3 & $\mathrm{~L}$ \\
\hline Talaromyces & 2 & 1.90 & 2.22 & 2 & $\mathrm{~L}$ & & & & & & 2 & 1.63 & 2.22 & 2 & $\mathrm{~L}$ \\
\hline $\begin{array}{c}\text { T. duclauxii } \\
\text { (Delacr.) } \\
\text { Samson, N. } \\
\text { Yilmaz, Frisvad \& } \\
\text { Seifert } \\
\end{array}$ & 1 & 0.95 & 1.11 & 1 & $L$ & & & & & & 1 & 0.81 & 1.11 & 1 & $\mathrm{~L}$ \\
\hline $\begin{array}{l}\text { T. funiculosus } \\
\text { (Thom) Samson, } \\
\text { N. Yilmaz, } \\
\text { Frisvad \& Seifert }\end{array}$ & 1 & 0.95 & 1.11 & 1 & $\mathrm{~L}$ & & & & & & & & & & \\
\hline $\begin{array}{c}T . \\
\text { purpureogenus } \\
\text { Stoll }\end{array}$ & & & & & & & & & & & 1 & 0.81 & 1.11 & 1 & $\mathrm{~L}$ \\
\hline Trichoderma Sp. & 2 & 1.90 & 2.22 & 2 & $\mathrm{~L}$ & & & & & & & & & & \\
\hline $\begin{array}{c}\text { White Sterile } \\
\text { mycelium }\end{array}$ & 3 & 2.86 & 3.33 & 3 & $\mathrm{~L}$ & & & & & & & & & & \\
\hline Total count & 105 & 100 & 113.33 & - & - & 95 & 100 & 105.56 & - & - & 123 & 100 & 136.67 & - & - \\
\hline No. of genera & $10+1$ & 9 & 10 & & & & & & & & & & & & \\
\hline No. of species & $19+1$ & 18 & 17 & & & & & & & & & & & & \\
\hline
\end{tabular}

* OR=occurrence remark: $H=h i g h$ occurrence, between 7-10 cases (out of 10); $M=$ moderate occurrence, 4-6 cases; $L=l o w$ occurrence, 1 3 cases; $R=$ rare occurrence only one

\subsection{Total flavonoids content}

The total flavonoids content (TFC) was determined by the colorimetric method using quercetin standard as reported (Hossain and Rahman, 2011). Typically, $0.5 \mathrm{~mL}$ of methanolic extract, or standard in methanol was mixed with $100 \mu \mathrm{L}$ of aluminum chloride (10\%), $100 \mu \mathrm{L}$ of sodium acetate (1\%) and $4.3 \mathrm{~mL}$ distilled water. After an incubation period of the mixture at room temperature for $30 \mathrm{~min}$, the absorbance was measured at $415 \mathrm{~nm}$ using a Cary 60, Agilent technologies, UV/VIS spectrophotometer. The TFC was calculated using a standard calibration curve of quercetin $(12.5-400 \mathrm{mg} / \mathrm{L})$. The TFC was expressed as quercetin equivalent (QE) per $\mathrm{g}$ of plant dry weight (mgQE/gDW). All determinations were performed in triplicate and the results were averaged.

\subsection{Total antioxidant capacity}

The total antioxidant capacity (TAC) of the extracts was evaluated by phosphomolybdenum method with minor modifications as recorded in the literature (Hossain and Rahman, 2011; Prieto et al., 1999). Firstly, $100 \mu \mathrm{L}$ of the extract was combined with equivolume of the reagent solution, $3 \mathrm{~mL}$, which consists of $0.6 \mathrm{M}$ sulfuric acid, 28 $\mathrm{mM}$ sodium phosphate, and $4 \mathrm{mM}$ ammonium molybdate in glass tube. Next, the tubes were capped and incubated in a water bath at $95^{\circ} \mathrm{C}$ for $90 \mathrm{~min}$. After the samples cooled to room temperature, the absorbance of the developed color was measured at $695 \mathrm{~nm}$ against a blank using a Cary 60, Agilent technologies, UV/VIS spectrophotometer. A typical reagent blank was carried out by mixing $100 \mu \mathrm{L}$ of methanol with $3 \mathrm{~mL}$ of the reagent solution and then follow the same procedure for the extract. The experiments were performed in triplicate. The TAC was expressed as mg ascorbic acid equivalent (AA.E) per gram dry weight (mgAA.E/gDW). The standard calibration curve was constructed by measuring the absorbance of different concentrations of ascorbic acid (1, $12.5,25,50,100,200$ and $400 \mathrm{mg} / \mathrm{L})$.

\subsection{Liquid chromatography-electrospray ionization tandem mass spectrometry}

Liquid chromatography-electrospray ionization-tandem mass spectrometry (LC-ESI-MS/MS) is one of the top choices for analysis of plant polyphenols in complex mixtures due to its high sensitivity and efficiency (Desta et al., 2017). Analyses of bioactive compounds isolated from the endophytic fungi of the artichoke were carried out with Triple Quadrupole LC-DAD-ESI-MS/MS, Agilent 
6420 Series, system which consisted of a capillary HPLC equipped with a binary pump solvent delivery, thermostatic column compartment, diode array detector (DAD), and a QQQ 6420 mass detector coupled with a pneumatic nebulizer-assisted electrospray interface (ESI). A ZORBAX Eclipse XDB-C18 column (150 mm× $4.6 \mathrm{~mm}$ i.d., particle size $5 \mu \mathrm{m}$ (Agilent Technologies) was used. The mobile phase consisted of acetonitrile (A) and water/formic acid (99/1, v/v) (B) with the gradient system. The flow was maintained at $0.8 \mathrm{~mL} / \mathrm{min}$; sample injection was $3 \mu \mathrm{L}$. Negative electrospray mode was used for ionization of molecules.

\section{Results and discussion}

\subsection{Endophytes analysis}

Endophytic organisms have received considerable attention since 1981 when Weber discovered their great abilities to protect their host against insect pests, pathogens and even domestic herbivores (Webber, 1981). Additionally, they are now considered as an outstanding source of bioactive natural products especially those which colonize medicinal plants (Arnold et al., 2000; Strobel and Daisy, 2003; Wang et al., 2006; Xuan, 2011). Because the lack information about the endophytic fungal biodiversity in Egypt, the present work was initiated to explore the endophytic fungal population in two types of Artichoke which are widely used as medicinal plants and food. And, evaluation of the explored endophytic fungi abilities to produce flavonoids, phenolics and antioxidant compounds. List of recovered endophytic fungi, their total count, colonization frequency and occurrence in each studied plant from each studied media are presented in Tables 1 and 2. A total of 35 endophytic fungal species belonging to 14 genera were explored from total of 540 apparently healthy segments out of 60 plant samples from two Artichoke studied types. The gross total counts of colonizing endophytic fungi obtained from Baladi artichoke fresh leaves was 323 cfu on three different studied media with the majority of $123 \mathrm{cfu}$ originating from cellulose medium while the French artichoke fresh leaves recorded $259 \mathrm{cfu}$.

Table 2. Total count (TC), percentage of total count (\%TC), percentage of colonization frequency (\%CF), number of cases of isolation ( $\mathrm{NCl}$; out of 10 samples), occurrence remark $\left(\mathrm{OR}^{*}\right)$, of endophytic fungi isolated from French Hyrious Artichoke fresh leaves per 90 segments on the three types of media (PDA, CZ, \& cellulose)

\begin{tabular}{|c|c|c|c|c|c|c|c|c|c|c|c|c|c|c|c|}
\hline Types of media & & & PDA & & & & & $\mathrm{CZ}$ & & & & & C & & \\
\hline Endophytic fungi & Tc & \%Tc & $\% \mathrm{CF}$ & $\mathrm{NCl}$ & OR & Tc & \%Tc & \%CF & $\mathrm{NCl}$ & OR & Tc & \%Tc & $\% \mathrm{CF}$ & $\mathrm{NCl}$ & OR \\
\hline Alternaria & 44 & 50.00 & 48.89 & 10 & $\mathrm{H}$ & 47 & 47.00 & 52.22 & 10 & $\mathrm{H}$ & 39 & 54.93 & 43.33 & 8 & $\mathrm{H}$ \\
\hline $\begin{array}{c}\text { A. alternata (Fr.) } \\
\text { Keissl }\end{array}$ & 44 & 50.00 & 48.89 & 10 & $\mathrm{H}$ & 45 & 47.00 & 50.00 & 10 & $\mathrm{H}$ & 39 & 54.93 & 43.33 & 8 & $\mathrm{H}$ \\
\hline $\begin{array}{c}\text { A. radicina } \\
\text { Meier, Drechsler } \\
\text { \& E.D. Eddy } \\
\end{array}$ & & & & & & 1 & 1.00 & 1.11 & 1 & $\mathrm{~L}$ & & & & & \\
\hline $\begin{array}{c}\text { A tenuissima } \\
\text { (Kunze)Wiltshire }\end{array}$ & & & & & & 1 & 1.00 & 1.11 & 1 & $\mathrm{~L}$ & & & & & \\
\hline Aspergillus & 5 & 5.68 & 5.56 & 4 & $\mathrm{M}$ & 5 & 5.00 & 5.56 & 4 & $\mathrm{M}$ & 4 & 5.63 & 4.44 & 3 & $\mathrm{~L}$ \\
\hline A. flavus Link & 1 & 1.14 & 1.11 & 1 & $\mathrm{~L}$ & & & & & & & & & & \\
\hline A. niger Tiegh & 4 & 4.55 & 4.44 & 3 & $\mathrm{~L}$ & 4 & 4.00 & 4.44 & 3 & $\mathrm{~L}$ & 4 & 5.63 & 4.44 & 3 & L \\
\hline A. terreus Thom & & & & & & 1 & 1.00 & 1.11 & 1 & $\mathrm{~L}$ & & & & & \\
\hline $\begin{array}{c}\text { Chaetomium } \\
\text { globosum Kunze }\end{array}$ & 6 & 6.82 & 6.67 & 4 & $\mathrm{M}$ & 7 & 7.00 & 7.78 & 4 & $M$ & 1 & 1.41 & 1.11 & 1 & L \\
\hline Cladosporium & & & & & & 2 & 2.00 & 2.22 & 2 & $\mathrm{~L}$ & & & & & \\
\hline $\begin{array}{c}\text { C. } \\
\text { cladosporioides } \\
\text { (Fresen.) G.A. de } \\
\text { Vries }\end{array}$ & & & & & & 1 & 1.00 & 1.11 & 1 & $\mathrm{~L}$ & & & & & \\
\hline $\begin{array}{c}\text { C. } \\
\text { sphaerospermum } \\
\text { Penz }\end{array}$ & & & & & & 1 & 1.00 & 1.11 & 1 & $\mathrm{~L}$ & & & & & \\
\hline $\begin{array}{l}\text { Epicoccum } \\
\text { nigrum Link }\end{array}$ & & & & & & & & & & & 1 & 1.41 & 1.11 & 1 & $L$ \\
\hline Fusarium & 1 & 1.14 & 1.11 & 1 & $\mathrm{~L}$ & & & & & & 2 & 2.82 & 2.22 & 1 & $\mathrm{~L}$ \\
\hline $\begin{array}{l}\text { F. napiforme } \\
\text { Marasas, P.E. } \\
\text { Nelson \& Rabie }\end{array}$ & 1 & 1.14 & 1.11 & 1 & $L$ & & & & & & 1 & 1.41 & 1.11 & 1 & $\mathrm{~L}$ \\
\hline $\begin{array}{l}\text { F. fujikuroi } \\
\text { Nirenberg }\end{array}$ & & & & & & & & & & & 1 & 1.41 & 1.11 & 1 & $\mathrm{~L}$ \\
\hline $\begin{array}{l}\text { Nigrospora } \\
\text { panici Zimm }\end{array}$ & & & & & & 12 & 12.00 & 13.33 & 6 & $M$ & & & & & \\
\hline
\end{tabular}




\begin{tabular}{|c|c|c|c|c|c|c|c|c|c|c|c|c|c|c|c|}
\hline Types of media & & & PDA & & & & & CZ & & & & & C & & \\
\hline Endophytic fungi & Tc & \%Tc & $\% \mathrm{CF}$ & $\mathrm{NCl}$ & OR & Tc & \%Tc & $\% \mathrm{CF}$ & $\mathrm{NCl}$ & OR & Tc & \%Tc & $\% \mathrm{CF}$ & $\mathrm{NCl}$ & OR \\
\hline Penicillium & & & & & & & & & & & 2 & 2.82 & 2.22 & 2 & $\mathrm{~L}$ \\
\hline $\begin{array}{l}\text { P. fasciculatum } \\
\text { Sommerf. }\end{array}$ & & & & & & & & & & & 1 & 1.41 & 1.11 & 1 & $\mathrm{~L}$ \\
\hline $\begin{array}{c}\text { p. glabrum } \\
\text { (Wehmer) } \\
\text { Westling }\end{array}$ & & & & & & & & & & & 1 & 1.41 & 1.11 & 1 & $L$ \\
\hline $\begin{array}{c}\text { Sarocladium } \\
\text { strictum (W. } \\
\text { Gams) Summerb }\end{array}$ & & & & & & & & & & & 1 & 1.41 & 1.11 & 1 & $\mathrm{~L}$ \\
\hline $\begin{array}{c}\text { Stachybotrys } \\
\text { chartarum } \\
\text { (Ehrenb.) S. } \\
\text { Hughes }\end{array}$ & 1 & 1.14 & 1.11 & 1 & $\mathrm{~L}$ & 1 & 1.00 & 1.11 & 1 & $\mathrm{~L}$ & & & & & \\
\hline Stemphylium & 26 & 29.55 & 23.33 & 9 & $\mathrm{H}$ & 23 & 23.00 & 25.56 & 8 & $\mathrm{H}$ & 21 & 25.35 & 20.00 & 6 & $M$ \\
\hline $\begin{array}{c}\text { S. botryosum } \\
\text { Wallr. }\end{array}$ & 20 & 22.73 & 22.22 & 8 & $\mathrm{H}$ & 22 & 22.00 & 24.44 & 7 & $\mathrm{H}$ & 3 & 4.23 & 3.33 & 3 & L \\
\hline $\begin{array}{l}\text { S. vesicarium } \\
\text { (Wallr.) E.G. } \\
\text { Simmons }\end{array}$ & 6 & 6.82 & 6.67 & 3 & $L$ & 1 & 1.00 & 1.11 & 1 & $\mathrm{~L}$ & 18 & 25.35 & 20.00 & 6 & $M$ \\
\hline $\begin{array}{c}\text { Talaromyces } \\
\text { funiculosus } \\
\text { (Thom) Samson, } \\
\text { N. Yilmaz, } \\
\text { Frisvad \& Seifert }\end{array}$ & 4 & 4.55 & 4.44 & 4 & $M$ & & & & & & & & & & \\
\hline Sterile mycelium & 1 & 1.14 & 1.11 & 1 & $\mathrm{~L}$ & 3 & 3.00 & 3.33 & 1 & $\mathrm{~L}$ & & & & & \\
\hline Total count & 88 & 100.00 & 96.67 & - & - & 100 & 100.00 & 111.11 & - & - & 71 & 100.00 & 78.89 & - & - \\
\hline No. of genera & $7+1$ & $7+1$ & 7 & & & & & & & & & & & & \\
\hline No. of species & $9+1$ & $12+1$ & 11 & & & & & & & & & & & & \\
\hline
\end{tabular}

* OR=occurrence remark: $\mathrm{H}=$ high occurrence, between 7-10 cases (out of 10 ); $M=$ moderate occurrence, 4-6 cases; $\mathrm{L}=$ low occurrence, 1-3 cases; $R=$ rare occurrence only one

The total counts and colonization frequencies of endophytes in this study almost were within the range of many results obtained previously from other plants (Fróhlich et al., 2000; Liu et al., 2010; Orachaipunlap et al., 2009; Shankar Naik et al., 2008). Our results referred that there is high species richness and diversity indices of endophytic filamentous fungi in the leaves of Baladi Artichoke as compared to its French rival. Total fungal counts and colonization frequencies of endophytic fungi from both tested plants were higher spectrum on PDA than other tested media. In this respect, Mohanta et al. (Mohanta et al., 2008) found maximum number of endophytes on PDA when they investigated endophytes from three ethno-medicinal plants of Similipal Biosphere Reserve, India. Variation in the total counts and colonization frequencies between the two types of Artichoke on three different types of media that might be due to differences in medium constituents and site-specific factor. Variety of media composition help different fungal endophytes to grow; Such as Alternaria. graminicola and Curvularia australiensis recovered one time on PDA medium only. Additionally, Alternaria atra, $A$. radicina and $A$. phragmospora recovered from GlucoseCZ medium only for one time while Alernaria mali and Sarocladium strictum recovered from Cellulose medium. Furthermore, higher spectrum of the number of the collected endophytic species isolated from the Egyptian Baladi artichoke were observed compared to the French Hyrious artichoke in all tested media as shown in Figure 1.

All taxa recovered from Artichoke were belonging to Ascomycetes, a large number of genera and species of fungal Ascomycetes are able to live endophytically in plants (Petrini, 1986; Saar et al., 2001). There is no clear correlation between these fungal group and host categories. Alternaria alternata was the most common species as fungal endophyte in leaves of Baladi and French Artichoke, it was recovered in high frequency of occurrence comprising range from 36 to $58 \mathrm{cfu}$ (colony forming unit) as total count matching range from 40 to $64.44 \%$ of colonization frequency. Genus Alternaria was frequently isolated from leaves Artichoke, this genus demonstrated as endophytes of many plants previously (Fernandes et al., 2015; Moharram et al., 2016; Roy, 2001). In addition, fungal endophytes are reported to be host specific at the same time several species can also be isolated from different host (Suryanarayanan et al., 2002).

These results strongly suggested that existence of some degree of host specificity among fungal endophytes. Such host specific endophytes (isolated from only one plant) have also been observed in the present study: Alternaria atra $(1.05 \%)$, A. cheiranthi $(1.90 \%)$, A. chlamydospora 
(1.90\%), A. graminicola (0.95\%), A. citri (0.95\%), A. mali (0.81\%), A. phragmospora (1.05.\%), Cladosporium herbarum (1.05\%), Curvularia australiensis australiensis (0.95\%), Penicillium citrinum (1.05 and 2.86\%) and Talaromyces duclauxii (0.95 and $0.81 \%)$ were represented as fungal endophytes in Baladi Artichoke leaves only while A. radicina (1\%), Nigrospora panici $(12 \%)$, Stachybotrys chartarum (1 and 1.54\%). were represented as fungal endophytes in French Artichoke leaves.

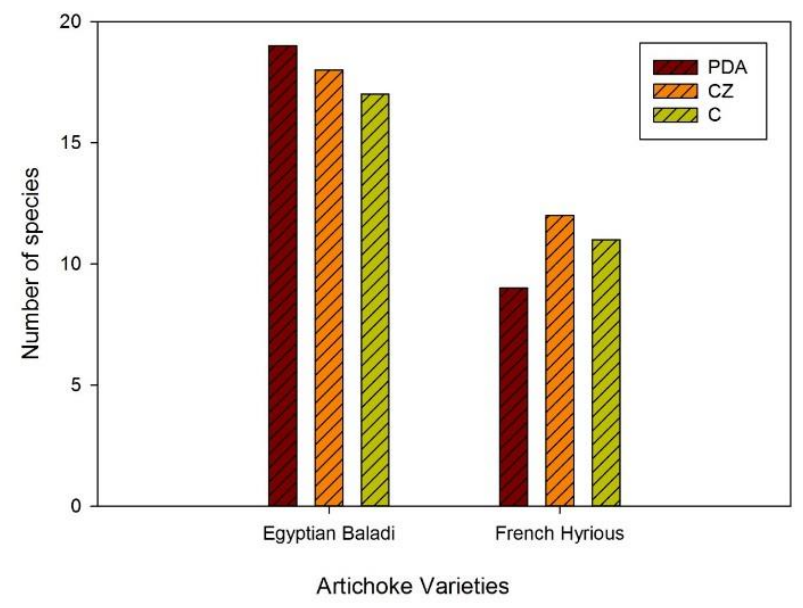

Figure 1. Distribution of endophytic fungi species isolated from the leaves of two varieties of Egyptian artichoke (French Hyrious and Egyptian Baladi) using three different types of tested media

(PDA, CZ, C)
3.2. Analysis of phenolic compounds and antioxidant activity

\subsubsection{Total phenolics content}

Natural phenolics are a major group of compounds acting as primary antioxidants and free radical scavengers (Benedec et al., 2012). Recently, they have attracted much interest due to their biological properties like anticancer, antimicrobial and antivirus activities (de Falco et al., 2015; Petropoulos et al., 2017; Simona Spînu et al., 2017; Zhou et al., 2016; Zhu et al., 2004). The TPC depends on the solvent used in the extraction method. For instance, Liu et al. proved that higher TPC was obtained for different extracts of endophyte using highly polar solvents (Liu et al., 2007). Thus, in our study, methanol was used as an extraction solvent due to it is the best solvent for the selectively of low and high molecular weight of phenolic compounds' extracts (Bimakr et al., 2011; Liu et al., 2007). In our study, the Egyptian Baladi Artichoke leaves of host plant contained $4.1 \mathrm{mg}$ GAE/g DW, whereas French Hyrious Artichoke leaves had $4.8 \mathrm{mg} \mathrm{GAE} / \mathrm{g} \mathrm{DW}$. Moreover, the TPC of the extract of endophytic fungi, belong to the Ascomycetes group isolated from the two leaves of Cynara scolymus $L$. artichoke; French Hyrious and Egyptian Baladi, was shown in Table 3.

Table 3. Total phenolics (TPC), total flavonoids (TFC) content and total antioxidant (TAC) as mg standard equivalent per g dry weight (mg standard/gDW)

\begin{tabular}{|c|c|c|c|c|c|c|}
\hline \multirow[b]{2}{*}{ Fungal species } & \multicolumn{3}{|c|}{ Egyptian Baladi Artichoke } & \multicolumn{3}{|c|}{ French Hyrious Artichoke } \\
\hline & TPC & TFC & TAC & TPC & TFC & TAC \\
\hline A. alternata & $32.41 \pm 0.01$ & $20.88 \pm 014$ & $310.09 \pm 0.29$ & $36.42 \pm 0.04$ & $25.63 \pm 3.23$ & $322.59 \pm 0.19$ \\
\hline A. cheiranthi & $20.38 \pm 0.03$ & $25.83 \pm 0.60$ & $268.50 \pm 35.11$ & & & \\
\hline A. chlamydospora & $12.88 \pm 0.01$ & $34.01 \pm 0.11$ & $207.20 \pm 0.20$ & & & \\
\hline A. graminicola & $29.72 \pm 0.25$ & $53.86 \pm 0.14$ & $357.03 \pm 0.27$ & & & \\
\hline A. radicina & & & & $24.91 \pm 0.03$ & $27.29 \pm 0.75$ & $278.67 \pm 0.17$ \\
\hline A. flavus & $31.98 \pm 0.03$ & $73.90 \pm 2.35$ & $314.97 \pm 0.45$ & $22.36 \pm 0.02$ & $69.31 \pm 0.38$ & $294.14 \pm 0.22$ \\
\hline A. niger & $21.06 \pm 0.01$ & $58.81 \pm 0.15$ & $193.55 \pm 0.34$ & $14.89 \pm 0.02$ & $43.38 \pm 0.11$ & $170.09 \pm 0.15$ \\
\hline Epicocum nigrum & $10.38 \pm 0.01$ & $132.71 \pm 0.31$ & $162.96 \pm 0.30$ & $19.48 \pm 0.02$ & $173.55 \pm 0.59$ & $203.33 \pm 0.20$ \\
\hline F. fujikuroi & $39.20 \pm 0.06$ & $132.03 \pm 1.27$ & $190.17 \pm 0.14$ & $33.19 \pm 0.06$ & $17.55 \pm 0.43$ & $244.06 \pm 0.39$ \\
\hline P. glabrum & $18.73 \pm 0.02$ & $29.65 \pm 0.05$ & $364.21 \pm 0.70$ & $23.98 \pm 0.13$ & $30.18 \pm 0.54$ & $308.69 \pm 0.49$ \\
\hline S. vesicarium & $17.33 \pm 0.04$ & $13.92 \pm 0.06$ & $306.55 \pm 1.10$ & $18.49 \pm 0.00$ & $13.43 \pm 0.77$ & $230.02 \pm 0.19$ \\
\hline Talaromyces sp. & $40.30 \pm 0.07$ & $76.67 \pm 1.25$ & $680.92 \pm 0.59$ & $40.01 \pm 0.10$ & $48.95 \pm 0.71$ & $399.96 \pm 0.06$ \\
\hline Sterile mycelium & $28.67 \pm 0.10$ & $29.65 \pm 0.05$ & $308.69 \pm 0.49$ & $26.08 \pm 0.04$ & $24.17 \pm 0.26$ & $206.94 \pm 6.70$ \\
\hline
\end{tabular}

All values expressed as mean of triplicate measurements of TPC, TFC, and TAC \pm standard deviation (S.D.)

The results revealed that TPCs of endophytic fungi were higher than those in its host plant. Also, a wide range of TPC in endophytic fungal extracts was observed, from 10.38 to $40.30 \mathrm{mg} \mathrm{GAE} / \mathrm{g} \mathrm{DW}$, and 14.89 to $40.01 \mathrm{mg}$ GAE/g DW for the Egyptian Baladi and French Hyrious, respectively. The highest concentration of total phenolics was observed in Talaromyces $s p$. isolated from both Egyptian Baladi, and French Hyrious Artichoke leaves' extract to be $40.30 \mathrm{mg} \mathrm{GAE} / \mathrm{g} \mathrm{DW}$, and $40.01 \mathrm{mg} \mathrm{GAE} / \mathrm{g}$ DW, respectively. On the other hand, the lowest TPC were found in case of Epicocum nigrum (10.38 mg GAE/g DW), and $A$. niger (14.89 $\mathrm{mg}$ GAE/g DW) isolated from the Egyptian Baladi and French Hyrious Artichoke leaves' respectively. Our results showed higher polyphenolic content in A. alternata, Fusarium and Sterile mycelium, Penicillium species and Aspergillus species compared to the reported studies (Eskandarighadikolaii et al., 2015; Fernandes et al., 2009; Huang et al., 2007; Nagda et al., 2017; Yadav et al., 2014). Whereas the TPC values of Aspergillus sp. were slightly lower than those observed in endophytic fungi isolated from Eugenia jambolana and Aegle marmelos (Patil et al., 2015; Yadav et al., 2014). 


\subsubsection{Total flavonoids content}

Flavonoids (or bioflavonoids) are a class of plant and fungus secondary metabolites which are one of the most important polyphenols. The total flavonoid contents (TFC) of the methanolic extracts of endophytic fungi are summarized in Table 3. The TFC values of the leaves' extract were found to be $68.30 \mathrm{mg} \mathrm{QE} / \mathrm{g} \mathrm{DW}$ and $76.21 \mathrm{mgQE} / \mathrm{gDW}$ for the Egyptian Baladi and French Hyrious, respectively. Also, the endophytic fungi showed different amounts of flavonoids ranging from 13.92 to $173.99 \mathrm{mg} \mathrm{QE} / \mathrm{g}$ DW. As indicated in Table 3, Epicocum nigrum had the highest amount of the TFC in both varieties of Artichoke plant. While, the results illustrated that S. vesicarium, in the Egyptian Baladi and French Hyrious, had the lowest values of the TFC. The TFC of Penicillium species and Aspergillus species that have been reported in the literature (Nagda et al., 2017) have higher values compared to that isolated from Artichoke. The results suggested that the isolated endophytic fungi contain high antioxidants from the two different Artichoke plants as they have higher TFC compared to their hosts.

\subsubsection{Total antioxidant capacity}

The phosphomolybdate method has been used to evaluate the total antioxidant capacity (TAC) of endophytic fungi extracts. This method is based on the reduction of $\mathrm{Mo}(\mathrm{VI})$ to $\mathrm{Mo}(\mathrm{V})$ in the presence of the extracts, and it forms a green-colored phosphomolybdenum (V) complex, which registers a maximum absorbance at $695 \mathrm{~nm}$ (Prieto et al., 1999). Table 3 lists the total antioxidant capacity (TAC) of the methanolic extracts of the endophytic fungi. The extracts from leaves of the Egyptian Baladi and French Hyrious showed antioxidant activity with the value of 109.8 and $84.8 \mathrm{mgAA} . \mathrm{E} / \mathrm{g} \mathrm{DW}$, respectively. On the other hand, the extracts of the endophytic fungi contain high amount of TAC compared to the leaves of the plant. For instance, in the Egyptian Baladi, the TAC values varied widely and ranged from 163 to $681 \mathrm{mgAA} . \mathrm{E} / \mathrm{g} \mathrm{DW}$, while in the French Hyrious ranged from 170 to $400 \mathrm{mgAA}$.E/g DW. Therefore, the endophytic extracts could demonstrate to be more effective in the reduction of $\mathrm{Mo}(\mathrm{VI})$ to $\mathrm{Mo}(\mathrm{V})$ comparing to that of the Artichoke leaves. It is clear from Table 3 that the samples which had low phenolics content, it had lower antioxidant activity and vice-versa. Thus, the results are in agreement with the literature that demonstrate the linear relation between the total phenolics and total antioxidants content (Sultana et al., 2007; Yadav et al., 2014). For example, the Epicocum nigrum isolated from the Egyptian Baladi artichoke has the lowest TPC (10.38 mg GAE/g DW) and hence had the lowest TAC value (162.96 mgAA.E/g DW). As well as, the TPC and TAC values of $A$. niger isolated from the French Hyrious artichoke were $14.89 \mathrm{mg}$ GAE/g DW and 170.09 mg AA.E/g DW, respectively. So, it is widely accepted that antioxidant activity is strongly linked to the concentration of total phenolics and flavonoids. In general, our results indicate that the total antioxidant capacity of the different endophytic fungi were highly correlated to the total phenolics, as shown in Figure 2, which is in agreement with the reported in the literature (Fernandes et al., 2009; Nath et al., 2012; Wang et al., 2003; Yadav et al., 2014). Also Figure 2 depicts a good correlation between the total phenolics content and total antioxidant capacity estimation with $R^{2}$ value of 0.764 thereby confirm the anticipated linear relation.

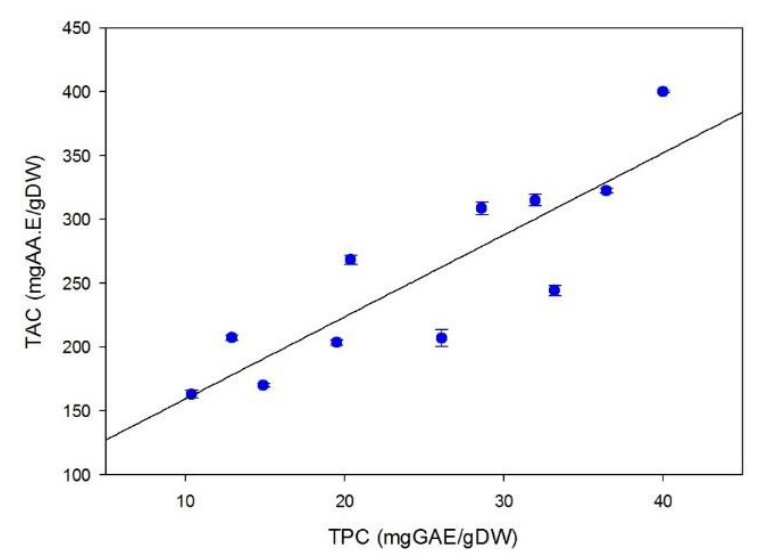

Figure 2. Correlation between TPC and TAC of the endophytic fungal extracts isolated from artichoke plant (French Hyrious and Egyptian Baladi)

\subsection{Identification of bioactive compounds isolated from endophytic fungi using HPLC-ESI-MS/MS}

Liquid chromatography-Electrospray Ionization-Tandem Mass Spectrometry (LC-ESI-MS/MS) is an extremely important analytical technique which is used for the separation, identification, and quantitation of an analyte of interest even if present in a complex mixture of different sample constituents (Abu-Reidah et al., 2015; Ramos et al., 2014). In the present work, a qualitative analysis of the bioactive compounds of the endophytic fungi isolated from the two varieties of artichoke (French Hyrious and Egyptian Baladi) has been carried out using HPLC-DAD-ESI-MS/MS in a negative ionization mode. Figure $3 A$ shows 5 peaks in the chromatogram of the phenolic compounds standard mixture at different retention time; 0.993, 1.347, 1.410, 1.704 and $1.997 \mathrm{~min}$, which are corresponding to caffeic acid, chlorogenic acid, 1,3-dicaffeoylquinic acid (1,3-diCQA), 1,5-dicaffeoylquinic acid (1,5-diCQA), and Luteolin-7-O-rutoniside, respecively. Furthermore, fragmentation of the standard mixture was performed using LC-ESI-MS/MS to confirm the molecular and precursor ions of each compound. For instance, the precursor ions [M-H] were found to be 179, 353, 515, 593 $\mathrm{m} / \mathrm{z}$ which are corresponding to caffeic acid $\left(\mathrm{C}_{9} \mathrm{H}_{8} \mathrm{O}_{4}\right)$, chlorogenic acid $\left(\mathrm{C}_{16} \mathrm{H}_{18} \mathrm{O}_{9}\right)$, dicaffeoylquinic acid $\left(\mathrm{C}_{25} \mathrm{H}_{23} \mathrm{O}_{12}\right)$, and Luteolin-7-O-rutoniside $\left(\mathrm{C}_{27} \mathrm{H}_{29} \mathrm{O}_{15}\right)$, respectively.

The fragmentation patterns of those compounds agree with the reported one (Abu-Reidah et al., 2012; Abu-Reidah et al., 2013; Andrei Fedosov et al., 2016; Chen et al., 2012; D'Antuono et al., 2015; Farag et al., 2013; Gebhardt, 1998; Gebhardt and Fausel, 1997; Llorach et al., 2002; Moglia et al., 2008; Sanchez-Rabaneda et al., 2003; Schutz et al., 2006; Sonnante et al., 2010; Wu et al., 2013). 
Moreover, identification of those constituents of the endophytic fungi, Alternaria alternata, isolated from the two varieties of artichoke was performed using LC, and LC-MS/MS. Figure 3B and $3 \mathrm{C}$ confirmed the presence of 1,3-diCQA and 1,5-diCQA (cynarin) in the endophytic fungi, Alternaria alternata, by comparing its retention time with the corresponding reference standards. Therefore, this novel study confirms the production of bioactive compounds from the endophytics fungi isolated from Artichoke. The obtained observations from this investigation are in matching with the compounds isolated from the artichoke plant as reported in the literature (Abu-Reidah et al., 2013; Farag et al., 2013). Thus, those endophytic fungus can be considered as potential source of antioxidants, anticancer, .... etc for further applications as well as good source for valuable products to produce from an easier and economic process.

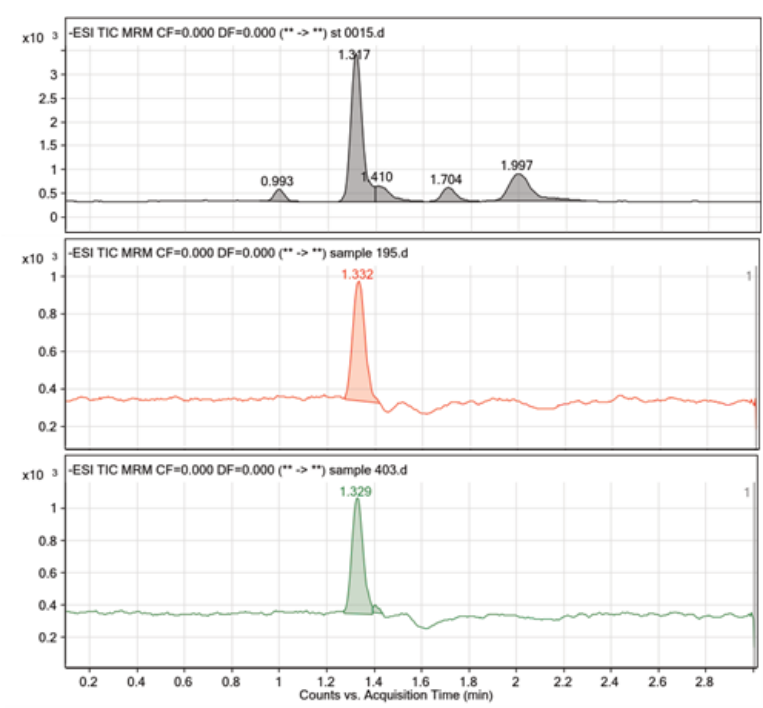

Figure 3. Total ion chromatograms (TIC) in negative mode of endophytic fungal methanolic extracts from two varieties of artichoke (French Hyrious and Egyptian Baladi)

\section{Conclusions}

This is the first study that reports the isolation and identification of the endophytic fungus from leaves of two varieties of Egyptian artichoke (French Hyrious and Egyptian Baladi) as well as the evaluation of their in vitro total antioxidant capacity (TAC), total phenolics content (TPC) and total flavonoids content (TFC). Significantly the endophytic fungi exhibited higher content of total phenolics, total flavonoids and antioxidant activity than their host plant leaves of Egyptian artichoke. Also, a total of 35 endophytic fungal species belonging to 14 genera were isolated from both artichoke leaves. It was found that Alternaria was the most frequently isolated genus and $A$. alternata was the most dominant species. All taxa recovered were assigned to Ascomycetes. The TAC, TPC and TFC of the fungal cultures ranged from 163 to 681 mgAA.E/g DW, 10.38 to $40.30 \mathrm{mgGAE} / \mathrm{gDW}$, and 13.92 to $173.99 \mathrm{mgQE} / \mathrm{gDW}$, respectively. Moreover, 1,3dicaffeoylquinic acid and 1,5-dicaffeoylquinic acid were identified in the methanolic extract of $A$. alternata using LC-ESI-MS/MS. In addition, this study demonstrated that the endophytic fungi have higher amount of bioactive compounds compared to that produced by its host plant. Therefore, the metabolites produced by the endophytic fungi isolated from the two varieties of Egyptian Artichoke may serve as an economic and potential source of natural antioxidants. Further investigations are needed to conform the identification of the isolated endophytic fungi.

\section{Acknowledgement}

The authors would like to thank the plant taxonomists; Prof. Dr. Abdel Aziz Fayed, Dr. Ahmed faried (Botany and Microbiology department, Assiut university) and Prof. Mohamed Ali Farghali (Department of Biological Sciences and Horticulture, Assiut University) for their help to identify the taxa of the Artichoke (Cynara scolymus L.) plants. Also, the authors thank the team of the Multidisciplinary Research Center of Excellence (MRCE) and the Analytical Chemistry Unit (ACAL) for their help to perform the analytical and bioanalytical studies. The author Abdulelah Thabet would like to thank Sana'a University, Yemen for the support.

\section{References}

Abu-Reidah I.M., Ali-Shtayeh M.S., Jamous R.M., Arraez-Roman D. and Segura-Carretero A. (2015), HPLC-DAD-ESI-MS/MS screening of bioactive components from Rhus coriaria $\mathrm{L}$. (Sumac) fruits, Food Chemistry, 166, 179-191. doi: 10.1016/j.foodchem.2014.06.011

Abu-Reidah I.M., Arráez-Román D., Quirantes-Piné R., Fernández-Arroyo S., Segura-Carretero A. and FernándezGutiérrez A. (2012), HPLC-ESI-Q-TOF-MS for a comprehensive characterization of bioactive phenolic compounds in cucumber whole fruit extract, Food Research International, 46(1), 108-117. doi: 10.1016/j.foodres.2011. 11.026

Abu-Reidah I.M., Arraez-Roman D., Segura-Carretero A. and Fernandez-Gutierrez A. (2013), Extensive characterisation of bioactive phenolic constituents from globe artichoke (Cynara scolymus L.) by HPLC-DAD-ESI-QTOF-MS, Food Chemistry, 141(3), 2269-2277. doi: 10.1016/j.foodchem.2013.04.066

Ainsworth E.A. and Gillespie K.M. (2007), Estimation of total phenolic content and other oxidation substrates in plant tissues using Folin-Ciocalteu reagent, Nature Protocols, 2(4), 875-877. doi: 10.1038/nprot.2007.102

Amita Pandey S.T. (2014), Concept of standardization, extraction and pre phytochemical screening strategies for herbal drug, Journal of Pharmacognosy and Phytochemistry, 2(5), 115-119.

Andrei Fedosov O.K., Gudzenko A., Semenchenko O. and Kyslychenko V. (2016), The determination of phenolic compounds in artichoke flower head extracts by hplc gc/ms technique, Scripta Scientifica Pharmaceutica, 3(2), 23-27.

Arnold A.E., Maynard Z., Gilbert G.S., Coley P.D. and Kursar T.A. (2000), Are tropical fungal endophytes hyperdiverse?, Ecology Letters, 3(4), 267-274. doi: doi:10.1046/j.14610248.2000.00159.x

Benedec D., Vlase L., Hanganu D. and Oniga I. (2012), Antioxidant Potential and Polyphenolic Content of Romanian 
Ocimum Basilicum, Digest Journal of Nanomaterials and Biostructures, 7(3), 1263-1270.

Bimakr M., Rahman R.A., Taip F.S., Ganjloo A., Salleh L.M., Selamat J., et al. (2011), Comparison of different extraction methods for the extraction of major bioactive flavonoid compounds from spearmint (Mentha spicata L.) leaves, Food and Bioproducts Processing, 89(1), 67-72. doi: 10.1016/j.fbp.2010.03.002

Chen H.J., Inbaraj B.S. and Chen B.H. (2012), Determination of phenolic acids and flavonoids in Taraxacum formosanum Kitam by liquid chromatography-tandem mass spectrometry coupled with a post-column derivatization technique, International Journal of Molecular Sciences, 13(1), 260-285. doi: 10.3390/ijms13010260

Chen X., Sang X., Li S., Zhang S. and Bai L. (2010), Studies on a chlorogenic acid-producing endophytic fungi isolated from Eucommia ulmoides Oliver, Journal of Industrial Microbiology and Biotechnology, 37(5), 447-454. doi: 10.1007/s10295-010-0690-0

Chen Y.-S., Cheng M.-J., Hsiao Y., Chan H.-Y., Hsieh S.-Y., Chang C.-W., Liu T.-W., Chang H.-S. and Chen I.-S. (2015), Chemical Constituents of the Endophytic Fungus Hypoxylon sp. 12F0687 Isolated from Taiwanese llex formosana, Helvetica Chimica Acta, 98(8), 1167-1176 doi: 10.1002/hlca.201500048.

Chutulo E.C. and Chalannavar R.K. (2018), Endophytic Mycoflora and Their Bioactive Compounds from Azadirachta Indica: A Comprehensive Review, Journal of Fungi (Basel), 4(2). doi: 10.3390/jof4020042

Dabbou S., Dabbou S., Pandino G., Arem A., Krimi K. and Helal A.N. (2017), Phenols and antioxidant properties of different parts of Tunisian globe artichoke heads, Journal of Bioresources Valorization, 2(1), 49-55.

D'Antuono I., Garbetta A., Linsalata V., Minervini F. and Cardinali A. (2015), Polyphenols from artichoke heads (Cynara cardunculus (L.) subsp. scolymus Hayek): in vitro bio-accessibility, intestinal uptake and bioavailability, Food and Function. doi: 10.1039/c5fo00137d

de Falco B., Incerti G., Amato M. and Lanzotti V. (2015), Artichoke: botanical, agronomical, phytochemical, and pharmacological overview, Phytochemistry Reviews, 14(6), 993-1018. doi: 10.1007/s11101-015-9428-y

Desta K.T., Kim G.S., Abd El-Aty A.M., Raha S., Kim M.B., Jeong J. et al. (2017), Flavone polyphenols dominate in Thymus schimperi Ronniger: LC-ESI-MS/MS characterization and study of anti-proliferative effects of plant extract on AGS and HepG2 cancer cells, Journal of chromatography. B, Analytical Technologies in the Biomedical and Life Sciences, 1053, 1-8. doi: 10.1016/j.jchromb.2017.03.035

El Morsy E.M. and Kamel R. (2014), Protective effect of artichoke leaf extract against paracetamol-induced hepatotoxicity in rats. Pharmaceutical Biology, 1-7. doi: 10.3109/13880209. 2014.913066

Eskandarighadikolaii S., Cruz T. and Bungihan M. (2015), Antioxidant properties of fungal endophytes associated with the three medicinal plants Gliricidia sepium, Canna indica and Gardenia jasminoides, Journal of Scientific Research and Reports, 6(3), 217-226. doi: 10.9734/jsrr/2015/16272

FAO. (2016), FAOSTAT, 2016. Food and Agricultural Commodities Production. Final 2016 Data. Retrieved from http://www.fao.org/faostat/en/\#rankings/countries_by_co mmodity

Farag M.A., El-Ahmady S.H., Elian F.S. and Wessjohann L.A. (2013), Metabolomics driven analysis of artichoke leaf and its commercial products via UHPLC-q-TOF-MS and chemometrics, Phytochemistry, 95, 177-187. doi: 10.1016/ j.phytochem.2013.07.003

Fernandes E.G., Pereira O.L., da Silva C.C., Bento C.B. and de Queiroz M.V. (2015), Diversity of endophytic fungi in Glycine max, Microbiological Research, 181, 84-92. doi: 10.1016/j.micres.2015.05.010

Fernandes M.d.R.V., Silva T.A.C.e., Pfenning L.H., Costa-Neto C.M.d., Heinrich T.A., Alencar S.M.d., et al. (2009), Biological activities of the fermentation extract of the endophytic fungus Alternaria alternata isolated from Coffea arabica $L$. Brazilian Journal of Pharmaceutical Sciences, 45, 677-685.

Fróhlich J., Hyde K.D. and Petrini O. (2000), Endophytic fungi associated with palms. Mycological Research, 104(10), 12021212. doi: https://doi.org/10.1017/S095375620000263X

Gebhardt R. (1998), Inhibition of cholesterol biosynthesis in primary cultured rat hepatocytes by artichoke (Cynara scolymus L.) extracts, Journal of Pharmacology and Experimental Therapeutics, 286(3), 1122-1128.

Gebhardt R. and Fausel M. (1997), Antioxidant and hepatoprotective effects of artichoke extracts and constituents in cultured rat hepatocytes, Toxicology in Vitro, 11(5), 669-672.

Gunatilaka A.A. (2006), Natural products from plant-associated microorganisms: distribution, structural diversity, bioactivity, and implications of their occurrence, Journal of Natural Products, 69(3), 509-526. doi: 10.1021/np058128n

Heidarian E. and Rafieian-Kopaei M. (2013), Protective effect of artichoke (Cynara scolymus) leaf extract against lead toxicity in rat, Pharmaceutical Biology, 51(9), 1104-1109. doi: 10.3109/13880209.2013.777931

Hossain M.A. and Rahman S.M.M. (2011), Total phenolics, flavonoids and antioxidant activity of tropical fruit pineapple, Food Research International, 44(3), 672-676. doi: 10.1016/ j.foodres.2010.11.036

Huang W.-Y., Cai Y.-Z., Hyde K.D., Corke H. and Sun M. (2007), Endophytic fungi from Nerium oleander L (Apocynaceae): main constituents and antioxidant activity, World Journal of Microbiology and Biotechnology, 23(9), 1253-1263. doi: 10.1007/s11274-007-9357-z

Jacociunas L.V., de Andrade H.H., Lehmann M., de Abreu B.R., Ferraz Ade B., da Silva J., et al. (2013), Artichoke induces genetic toxicity in the cytokinesis-block micronucleus (CBMN) cytome assay, Food and Chemical Toxicology, 55, 56-59. doi: 10.1016/j.fct.2012.12.024

Kusku-Kiraz Z., Mehmetcik G., Dogru-Abbasoglu S. and Uysal M. (2010), Artichoke leaf extract reduces oxidative stress and lipoprotein dyshomeostasis in rats fed on high cholesterol diet, Phytotherapy Research, 24(4), 565-570. doi: 10.1002/ptr.2985

Lattanzio V., Kroon P.A., Linsalata V. and Cardinali A. (2009), Globe artichoke: A functional food and source of nutraceutical ingredients, Journal of Functional Foods, 1(2), 131-144. doi: http://dx.doi.org/10.1016/j.jff.2009.01.002

Liang H., Xing Y., Chen J., Zhang D., Guo S. and Wang C. (2012), Antimicrobial activities of endophytic fungi isolated from Ophiopogon japonicus (Liliaceae), BMC Complementary and 
Alternative Medicine, 12, 238. doi: 10.1186/1472-6882-12238

Liu C., Liu T., Fengfeng Y. and Yucheng G.U. (2010), Full Length Research Paper Isolating endophytic fungi from evergreen plants and determining their antifungal activities, African Journal of Microbiology Research, 4(21), 2243-2248.

Liu X., Dong M., Chen X., Jiang M., Lv X. and Yan G. (2007), Antioxidant activity and phenolics of an endophytic Xylaria sp. from Ginkgo biloba, Food Chemistry, 105(2), 548-554. doi: 10.1016/j.foodchem.2007.04.008

Llorach R., Espin J.C., Tomas-Barberan F.A. and Ferreres F. (2002), Artichoke (Cynara scolymus L.) byproducts as a potential source of health-promoting antioxidant phenolics, Journal of Agricultural and Food Chemistry, 50(12), 34583464.

Moglia A., Lanteri S., Comino C., Acquadro A., de Vos R. and Beekwilder J. (2008), Stress-induced biosynthesis of dicaffeoylquinic acids in globe artichoke, Journal of Agricultural and Food Chemistry, 56(18), 8641-8649. doi: 10.1021/jf801653w

Mohanta J., Tayung K. and Mohapatra U. (2008), Antimicrobial potentials of endophytic fungi inhabiting three ethnomedicinal plants of Similipal Biosphere Reserve, India, Internet Journal of Microbiology, 5(2).

Moharram A., Zohri A.-N. and Seddek N. (2016), L-Asparaginase Production by Endophytic Fungi Isolated from Withania Somnifera in Egypt (Vol. 2).

Nagda V., Gajbhiye A. and Chhatwani D.K. (2017), Isolation and characterization of endophytic fungi from calotropis procera for their antioxidant activity, Asian Journal of Pharmaceutical and Clinical Research, 10(3), 254. doi: 10.22159/ajpcr.2017.v10i3.16125

Nassar M.I., Mohamed T.K., Elshamy A.I., El-Toumy S.A., Abdel Lateef A.M. and Farrag A.R. (2013), Chemical constituents and anti-ulcerogenic potential of the scales of Cynara scolymus (artichoke) heads, Journal of the Science of Food and Agriculture, 93(10), 2494-2501. doi: 10.1002/jsfa.6065

Nath A., Raghunatha P. and Joshi S.R. (2012), Diversity and Biological Activities of Endophytic Fungi of Emblica officinalis, an Ethnomedicinal Plant of India, Mycobiology, 40(1), 8-13. doi: 10.5941/MYCO.2012.40.1.008

Ncube N.S., Afolayan A.J. and Anthony I.O. (2008), Assessment techniques of antimicrobial properties of natural compounds of plant origin: current methods and future trends, African Journal of Biotechnology, 7(12), 1797-1806. doi: 10.5897/AJB07.613

Orachaipunlap K., Roengsumran S. and Sihanonth P. (2009), Diversity of Endophytic Fungi Isolated From Plant Leaves of Deciduous Dipterocarp Forest in Tak Province (Vol. 43).

Pandey A.K., Reddy M.S.a. and Suryanarayanan T.S. (2003). ITSRFLP and ITS sequence analysis of a foliar endophytic Phyllosticta from different tropical trees, Mycological Research, 107(4), 439-444. doi: 10.1017/s0953756203007494

Patil M.P., Patil R.H. and Maheshwari V.L. (2015), Biological activities and identification of bioactive metabolite from endophytic aspergillus flavus $\mathrm{L7}$ isolated from aegle marmelos. Current Microbiology, 71(1), 39-48. doi: 10.1007/s00284-015-0805-y

Petrini O. (1986), Taxonomy of endophytic fungi of aerial plant tissues.
Petropoulos S.A., Pereira C., Barros L. and Ferreira I. (2017), Leaf parts from Greek artichoke genotypes as a good source of bioactive compounds and antioxidants, Food and Function, 8(5), 2022-2029. doi: 10.1039/c7fo00356k

Prieto P., Pineda M. and Aguilar M. (1999), Spectrophotometric quantitation of antioxidant capacity through the formation of a phosphomolybdenum complex: Specific application to the determination of vitamin E, Analytical Biochemistry, 269(2), 337-341. doi: http://dx.doi.org/10.1006/abio.1999. 4019

Ramos P.A.B., Santos S.A.O., Guerra Â.R., Guerreiro O., Freire C.S.R., Rocha S.M., et al. (2014), Phenolic composition and antioxidant activity of different morphological parts of Cynara cardunculus L. var. altilis (DC), Industrial Crops and Products, 61(0), 460-471. doi: http://dx.doi.org/ 10.1016/ j.indcrop.2014.07.042

Roghani-Dehkordi F. and Kamkhah A.F. (2009), Artichoke leaf juice contains antihypertensive effect in patients with mild hypertension, Journal of Dietary Supplements, 6(4), 328-341. doi: 10.3109/19390210903280207

Roy K.W., Baird R.E. and Abney T.S. (2001), A review of soybean (Glycine max) seed, pod, and flowermycofloras in North America, with methods and key for identification of selected fungi, Mycopathologia, 150(1), 15-27.

Ruiz-Cano D., Perez-Llamas F., Frutos M.J., Arnao M.B., Espinosa C., Lopez-Jimenez J.A., et al. (2014), Chemical and functional properties of the different by-products of artichoke (Cynara scolymus L.) from industrial canning processing, Food Chemistry, 160, 134-140. doi: 10.1016/j.foodchem.2014.03. 091

Saar D.E., Polans N.O., Sørensen P.D. and Duvall M.R. (2001), Angiosperm DNA contamination by endophytic fungi: Detection and methods of avoidance, Plant Molecular Biology Reporter, 19(3), 249-260. doi: 10.1007/bf02772897

Saez V., Fasoli E., D'Amato A., Simo-Alfonso E. and Righetti P.G. (2013), Artichoke and cynar liqueur: two (not quite) entangled proteomes, Biochimica et Biophysica Acta, 1834(1), 119-126. doi: 10.1016/j.bbapap.2012.08.020

Sanchez-Rabaneda F., Jauregui O., Lamuela-Raventos R.M., Bastida J., Viladomat F. and Codina C. (2003), Identification of phenolic compounds in artichoke waste by highperformance liquid chromatography-tandem mass spectrometry, Journal of Chromatography A, 1008(1), 57-72.

Sandhu S. and Gupta D. (2015), Role of endophytic fungi in preservation of plant biodiversity, International Journal of advances in Pharmacy, Biology and Chemistry, 4(3), 635-647.

Schulz B., Boyle C., Draeger S., Römmert A.-K. and Krohn K. (2002), Endophytic fungi: a source of novel biologically active secondary metabolites, Mycological Research, 106(9), 9961004. doi: 10.1017/s0953756202006342.

Schulz B., Wanke U., Draeger S. and Aust H.J. (1993), Endophytes from herbaceous plants and shrubs: effectiveness of surface sterilization methods, Mycological Research, 97(12), 14471450. doi: http://dx.doi.org/10.1016/S0953-7562(09)802153.

Schutz K., Muks E., Carle R. and Schieber A. (2006), Quantitative determination of phenolic compounds in artichoke-based dietary supplements and pharmaceuticals by highperformance liquid chromatography, Journal of Agricultural and Food Chemistry, 54(23), 8812-8817. doi: 10.1021/jf062009b. 
Shankar Naik B., Shashikala J. and Krishnamurthy Y.L. (2008), Diversity of fungal endophytes in shrubby medicinal plants of Malnad region, Western Ghats, Southern India, Fungal Ecology, 1(2), 89-93. doi: https://doi.org/10.1016/j.funeco. 2008.05.001

Sharma D., Pramanik A. and Agrawal P.K. (2016), Evaluation of bioactive secondary metabolites from endophytic fungus Pestalotiopsis neglecta BAB-5510 isolated from leaves of Cupressus torulosa D.Don, 3 Biotech, 6(2), 210. doi: 10.1007/s13205-016-0518-3

Silva-Hughes A.F., Wedge D.E., Cantrell C.L., Carvalho C.R., Pan Z., Moraes R.M., et al. (2015), Diversity and antifungal activity of the endophytic fungi associated with the native medicinal cactus Opuntia humifusa (Cactaceae) from the United States, Microbiological Research, 175, 67-77. doi: 10.1016/j.micres.2015.03.007

Smith D. and Onions A.H.S. (1994), Preservation and Maintenance of Living Fungi (2nd ed. Vol. 5 (4)). CAB International, Wallingford.

Sonnante G., D'Amore R., Blanco E., Pierri C.L., De Palma M., Luo J., et al. (2010), Novel hydroxycinnamoyl-coenzyme A quinate transferase genes from artichoke are involved in the synthesis of chlorogenic acid, Plant Physiology, 153(3), 12241238. doi: 10.1104/pp.109.150144

Spînu S., Ionescu D., Drugulescu M., Orţan A., Morar I. and Turtoi M. (2017), Biological aspects, composition and potential application of cynara scolymus L., Current Trends in Natural Sciences, 6(11), 98-104.

Stierle A., Strobel G., Stierle D., Grothaus P. and Bignami G. (1995), The search for a taxol-producing microorganism among the endophytic fungi of the Pacific yew, Taxus brevifolia, Journal of Natural Products, 58(9), 1315-1324.

Strobel G. and Daisy B. (2003), Bioprospecting for Microbial Endophytes and Their Natural Products, Microbiology and Molecular Biology Reviews, 67(4), 491-502. doi: 10.1128/mmbr.67.4.491-502.2003

Sultana B., Anwar F. and Przybylski R. (2007), Antioxidant activity of phenolic components present in barks of Azadirachta indica, Terminalia arjuna, Acacia nilotica, and Eugenia jambolana Lam. Trees, Food Chemistry, 104(3), 1106-1114. doi: 10.1016/j.foodchem.2007.01.019

Suryanarayanan T.S., Venkatesan G. and Murali T.S. (2003), Endophytic fungal communities in leaves of tropical forest trees: Diversity and distribution patterns, Current Science, 85(4), 489-492.

Sutjaritvorakul T., Whalley A.J.S., Sihanonth P., Roengsumran S. (2011), Antimicrobial activity from endophytic fungi isolated from plant leaves in Dipterocarpous forest at Viengsa district Nan province, Thailand, Journal of Agricultural Technology, 7(1), 115-121.

Wang F.W., Jiao R.H., Cheng A.B., Tan S.H. and Song Y.C. (2006), Antimicrobial potentials of endophytic fungi residing in Quercus variabilis and brefeldin A obtained from Cladosporium sp., World Journal of Microbiology and Biotechnology, 23(1), 79-83. doi: 10.1007/s11274-006-91954

Wang K.W., Wang S.W., Wu B. and Wei J.G. (2014), Bioactive natural compounds from the mangrove endophytic fungi, Mini Reviews in Medicinal Chemistry, 14(4), 370-391.

Wang M., Simon J.E., Aviles I.F., He K., Zheng Q.Y. and Tadmor Y. (2003), Analysis of antioxidative phenolic compounds in artichoke (Cynara scolymus L.), Journal of Agricultural and Food Chemistry, 51(3), 601-608. doi: 10.1021/jf020792b

Wang X., Radwan M.M., Tarawneh A.H., Gao J., Wedge D.E., Rosa L.H., et al. (2013), Antifungal activity against plant pathogens of metabolites from the endophytic fungus Cladosporium cladosporioides, Journal of Agricultural and Food Chemistry, 61(19), 4551-4555. doi: 10.1021/jf400212y

Webber J. (1981), A natural biological control of Dutch elm disease, Nature, 292, 449. doi: 10.1038/292449a0

Wojdylo A., Oszmianski J. and Czemerys R. (2007), Antioxidant activity and phenolic compounds in 32 selected herbs, Food Chemistry, 105(3), 940-949. doi: 10.1016/ j.foodchem.2007.04.038

Wu J., Qian Y., Mao P., Chen L., Lu Y. and Wang H. (2013), Separation and identification of phenolic compounds in canned artichoke by LC/DAD/ESI-MS using core-shell C18 column: a comparative study. Journal of Chromatography. B, Analytical Technologies in the Biomedical and Life Sciences, 927, 173-180. doi: 10.1016/j.jchromb.2012.11.026

Xia N., Pautz A., Wollscheid U., Reifenberg G., Forstermann U. and $\mathrm{Li} \mathrm{H.} \mathrm{(2014),} \mathrm{Artichoke,} \mathrm{cynarin} \mathrm{and} \mathrm{cyanidin}$ downregulate the expression of inducible nitric oxide synthase in human coronary smooth muscle cells, Molecules, 19(3), 3654-3668. doi: 10.3390/molecules19033654

Xuan Q.B., F.K., Pan H.M. and Liu A.H. (2011), Isolating fungal endophyte from Paris polyphylla Smith var. yunnanensis and dentifying their antibacterial ability, African Journal of Microbiology Research, 4(10), 1001-1004.

Yadav M., Yadav A. and Yadav J.P. (2014), In vitro antioxidant activity and total phenolic content of endophytic fungi isolated from Eugenia jambolana Lam, Asian Pacific Journal of Tropical Medicine, 7S1, S256-S261. doi: 10.1016/S19957645(14)60242-X.

Yoo K.S., Lee E.J., Leskovar D. and Patil B.S. (2012), Development of an automated method for Folin-Ciocalteu total phenolic assay in artichoke extracts, Journal of Food Science, 77(12), C1279-1284. doi: 10.1111/j.1750-3841.2012.02973.x

Zhou Y., Zheng J., Li Y., Xu D.P., Li S., Chen Y.M. and Li H.B. (2016), Natural polyphenols for prevention and treatment of cancer, Nutrients, 8(8). doi: 10.3390/nu8080515

Zhu X., Zhang H. and Lo R. (2004), Phenolic compounds from the leaf extract of artichoke (Cynara scolymus L.) and their antimicrobial activities, Journal of Agricultural and Food Chemistry, 52(24), 7272-7278. 